08

\title{
Исследование оптических и структурных свойств трехмерных островков InGaP(As), сформированных методом замещения элементов пятой группы
}

\author{
(C) А.Г. Гладышев, ${ }^{1}$ А.В. Бабичев, ${ }^{1}$ В.В. Андрюшкин, ${ }^{1}$ Д.В. Денисов, ${ }^{2}$ В.Н. Неведомский, ${ }^{3}$ Е.С. Колодезный, ${ }^{1}$ \\ И.И. Новиков, ${ }^{1}$ Л.Я. Карачинский, ${ }^{1}$ А.Ю. Егоров ${ }^{4}$ \\ ${ }^{1}$ Национальный исследовательский университет ИТМО РАН, \\ 197101 Санкт-Петербург, Россия \\ ${ }^{2}$ Санкт-Петербургский государственный электротехнический университет „ЛЭТИ“ им. В.И. Ульянова (Ленина), \\ 197376 Санкт-Петербург, Россия \\ ${ }^{3}$ Физико-технический институт им. А.Ф. Иоффре РАН, ЦКП „Материаловедение и диагностика в передовых технологиях“, \\ 194021 Санкт-Петербург, Россия \\ ${ }^{4}$ ООО „Коннектор Оптикс“, \\ 194292 Санкт-Петербург, Россия \\ e-mail: glad@mail.ioffe.ru
}

Поступило в Редакцию 13 апреля 2020 г.

В окончательной редакции 12 мая 2020 г.

Принято к публикации 13 мая 2020 г.

Предложен новый метод формирования трехмерных квантово-размерных островков $\mathrm{InGaP}(\mathrm{As})$, заключающийся в замещении фосфора на мышьяк в слое InGaP, осажденном на GaAs непосредственно в процессе эпитаксиального роста. Показано, что при замещении фосфора на мышьяк в тонком слое InGaP формируются трехмерные островки, излучающие в спектральном диапазоне 0.95-0.97 $\mathrm{m}$ при комнатной температуре. Оценочная плотность островков составила $1.3 \cdot 10^{10} \mathrm{~cm}^{-2}$.

Ключевые слова: эпитаксия, квантовые точки, арсенид галлия, замещение фосфора, просвечивающая электронная микроскопия.

DOI: 10.21883/JTF.2020.12.50133.129-20

\section{Введение}

В настоящее время большой интерес проявляется к созданию приборов для управления одиночными фотонами [1-9] и нейроморфных вычислений, использующих в качестве излучателей полупроводниковые гетероструктуры с пониженной размерностью [10-12]. Наилучшими кандидатами на роль активных областей для излучателей в таких системах являются квантовые точки (КТ). Однако в отличие от традиционных лазерных применений, в которых массивы КТ должны иметь максимально возможную плотность [13-17], к активным областям в указанных выше системам предъявляется противоположное требование - уменьшение плотности КТ ниже значений $1 \cdot 10^{10} \mathrm{~cm}^{-2}[18,19]$. Для уменьшения плотности КТ используют специальные технологические режимы эпитаксиального роста, позволившие уменьшить плотность KT InAs с типичных $5 \cdot 10^{10} \mathrm{~cm}^{-2}$ до $\sim 1 \cdot 10^{9} \mathrm{~cm}^{-2}$ [20].

В настоящей работе предложен новый метод формирования трехмерных квантово-размерных островков путем замещения фосфора на мышьяк в слое InGaP, осажденном на GaAs непосредственно в процессе эпитаксиального роста. Были проведены ростовые эксперименты с различными технологическими режимами, исследованы оптические и структурные свойства полученных объектов.

\section{1. Эксперимент}

Гетероструктуры были изготовлены на полуизолирующих подложках $\mathrm{GaAs}$ диаметром $76.2 \mathrm{~mm}$ методом молекулярно-пучковой эпитаксии (МПЭ) на установке Riber49. Структуры состояли из буфера GaAs толщиной $100 \mathrm{~nm}$, обкладки GaAs толщиной $200 \mathrm{~nm}$, ограниченной со стороны подложки и поверхности барьерами AlGaAs толщиной по $100 \mathrm{~nm}$. Верхний барьер был прикрыт слоем GaAs толщиной $5 \mathrm{~nm}$ для предотвращения окисления. В центр обкладки помещался слой InGaP, решеточно-согласованный с подложкой. Все структуры выращивались при температуре $580^{\circ} \mathrm{C}$. Слой $\mathrm{InGaP}$, а также прикрывающий его слой GaAs толщиной $5 \mathrm{~nm}$ осаждались при температуре $520^{\circ} \mathrm{C}$. Трехмерные островки формировались следующим образом. После осаждения слоя InGaP в потоке фосфора происходила остановка роста и замена потока фосфора на поток мышьяка в течение $30 \mathrm{~s}$. Затем происходила выдержка ростовой поверхности исключительно в потоке мышьяка в течение 5-10 min, во время которой происходило замещение атомов фосфора в слое InGaP на атомы мышьяка с последующим формированием трехмерных островков $\mathrm{InGaP}(\mathrm{As})$. После этого слой $\mathrm{InGaP}(\mathrm{As})$ заращивался $5 \mathrm{~nm}$ GaAs, затем происходила остановка роста и подъем температуры подложки до $580^{\circ} \mathrm{C}$ в тече- 
Особенности технологических режимов формирования образцов $\mathrm{C} 1-\mathrm{C} 4$

\begin{tabular}{c|c|c|c}
\hline № структуры & Толщина слоя InGaP, nm & Время выдержки, min & Температура подложки во время выдержки, ${ }^{\circ} \mathrm{C}$ \\
\hline $\mathrm{C} 1$ & 2 & 5 & 520 \\
\hline $\mathrm{C} 2$ & 2 & $5(0.5+4.5)$ & 535 \\
\hline $\mathrm{C} 3$ & 3 & $10(0.5+9.5)$ & 535 \\
\hline С4 (для ПЭМ) & 3 & $10(0.5+9.5)$ & 535
\end{tabular}

ние $3 \mathrm{~min}$. После этого ростовой процесс возобновлялся. Процесс формирования островков $\operatorname{InGaP}(\mathrm{As})$ контролировался по изменению картины дифракции быстрых электронов на ростовой поверхности во время выдержки в потоке мышьяка.

На ростовой поверхности в используемых в настоящей работе режимах роста постоянно происходят процессы адсорбции и десорбции атомов. Это в большей степени относится к элементам пятой группы. Заменив поток фосфора на поток мышьяка, становится возможным преобразовать слой $\mathrm{InGaP}$ в $\operatorname{InGaP}(\mathrm{As})$ за счет десорбции атомов фосфора и адсорбции вместо них атомов мышьяка. По мере увеличения концентрации мышьяка в слое $\operatorname{InGaP}(\mathrm{As})$ увеличивалась и его постоянная решетки, что приводило к возникновению растущего механического напряжения в преобразуемом слое. После превышения критической величины напряжения происходила его релаксация путем формирования трехмерных островков.

Была изготовлена серия образцов $(\mathrm{C} 1-\mathrm{C} 4)$, отличающихся толщиной слоя изначального InGaP, температурой подложки во время выдержки и длительностью выдержки в потоке мышьяка. Особенности технологических режимов формирования образцов представлены в таблице.

Структура С4 была изготовлена специально для исследования методом просвечивающей электронной микроскопии (ПЭМ) и являлась полным аналогом структуры C3, но слой $\operatorname{InGaP}(\mathrm{As})$ в ней был прикрыт только слоем GaAs толщиной $25 \mathrm{~nm}$. В образцах $\mathrm{C} 2-\mathrm{C} 4$ после осаждения слоя InGaP в течение $30 \mathrm{~s}$ после смены потоков элементов пятой группы происходило повышение температуры подложки на $15^{\circ} \mathrm{C}$.

Bсе структуры были исследованы методом фотолюминесценции (ФЛ) при комнатной температуре на установке RPM2000 Nanometrics. Возбуждение ФЛ производилось YAG: Nd лазером с длиной волны $532 \mathrm{~nm}$ и мощностью $45 \mathrm{~mW}$. Для оценки плотности трехмерных островков использовалась ПЭМ. Электронномикроскопические исследования выполнены с использованием оборудования ЦКП „Материаловедение и диагностика в передовых технологиях“ (ФТИ им. А.Ф. Иоффе, Санкт-Петербург) на микроскопе JEM-2100F при ускоряющем напряжении $200 \mathrm{kV}$. Для подготовки проб в планарной геометрии использовалась общепринятая методика механического утонения с распылением ионами $\mathrm{Ar}^{+}$на финишном этапе.

\section{2. Результаты и их обсуждение}

При изготовлении образца С1 до и после осаждения слоя $\mathrm{InGaP}$ от ростовой поверхности наблюдалась линейная картина дифракции с рефлексами $(4 \times 2)$, характерная для планарного роста в условиях, стабилизированных по элементу пятой группы. После начала выдержки поверхности слоя InGaP в потоке мышьяка картина дифракции начинала изменяться, на линейных рефлексах стали появляться утолщения, свидетельствующие о формировании трехмерных островков. Яркость и четкость наблюдаемых утолщений достигали максимума примерно через $3 \mathrm{~min}$ выдержки и затем слабо менялись. После окончания выдержки и осаждения $5 \mathrm{~nm}$ низкотемпературного GaAs картина дифракции вновь становилась линейной, что указывало на планаризацию ростовой поверхности. В процессе дальнейшего роста картина дифракции не изменялась. Для стимулирования процесса замещения фосфора на мышьяк в образце С2 была повышена температура подложки во время выдержки. Однако это не привело к принципиальным изменениям в картине дифракции. Для усиления наблюдаемых эффектов за счет возрастания механических напряжений в слое InGaP в образце C3 толщина слоя InGaP была увеличена до $3 \mathrm{~nm}$. Также было увеличено время выдержки до $10 \mathrm{~min}$. Введенные изменения привели к изменению наблюдаемой картины дифракции. Утолщения появились через $30 \mathrm{~s}$ и достигли максимальной яркости через $2 \mathrm{~min}$. После осаждения прикрывающего слоя GaAs утолщения сохранились, стали практически неразличимы только после осаждения примерно $10 \mathrm{~nm}$ GaAs на стандартной ростовой температуре.

На рис. 1 показаны спектры ФЛ структуры C1, измеренные в трех различных точках: у базового среза подложки (на удалении $30 \mathrm{~mm}$ от центра пластины), в центральной точке и у противоположного базовому срезу края (на удалении $30 \mathrm{~mm}$ от центра пластины). Видно, что на спектре помимо пиков от GaAs присутствует пик в области $970 \mathrm{~nm}$, соответствующий излучению от трехмерных островков $\mathrm{InGaP}(\mathrm{As})$. Положение пика меняется в пределах 968-980 nm. Интенсивность пика ФЛ при смещении от базового края к противоположному краю 


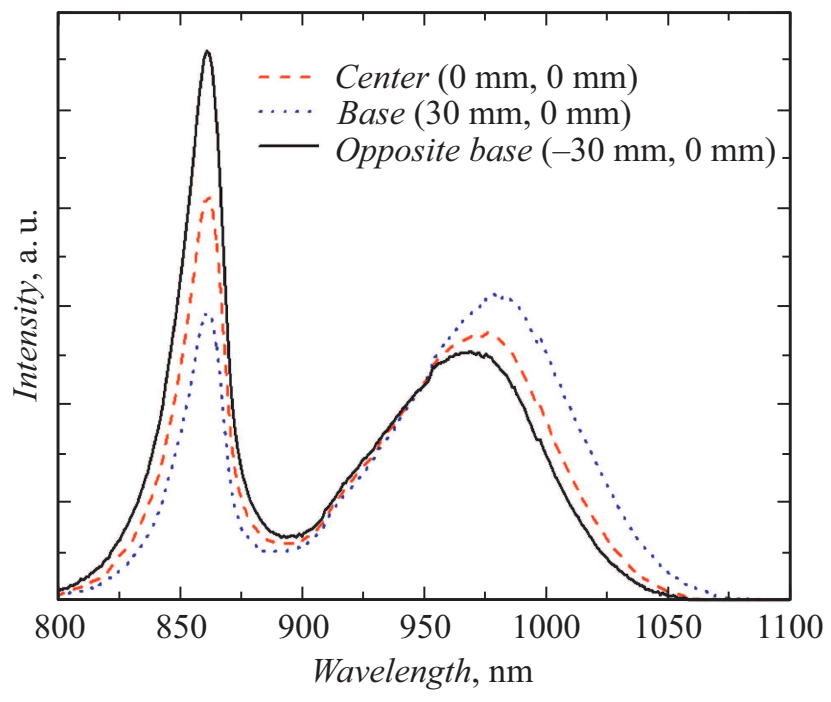

Рис. 1. Спектры ФЛ образца $\mathrm{C} 1$, измеренные в различных точках пластины.

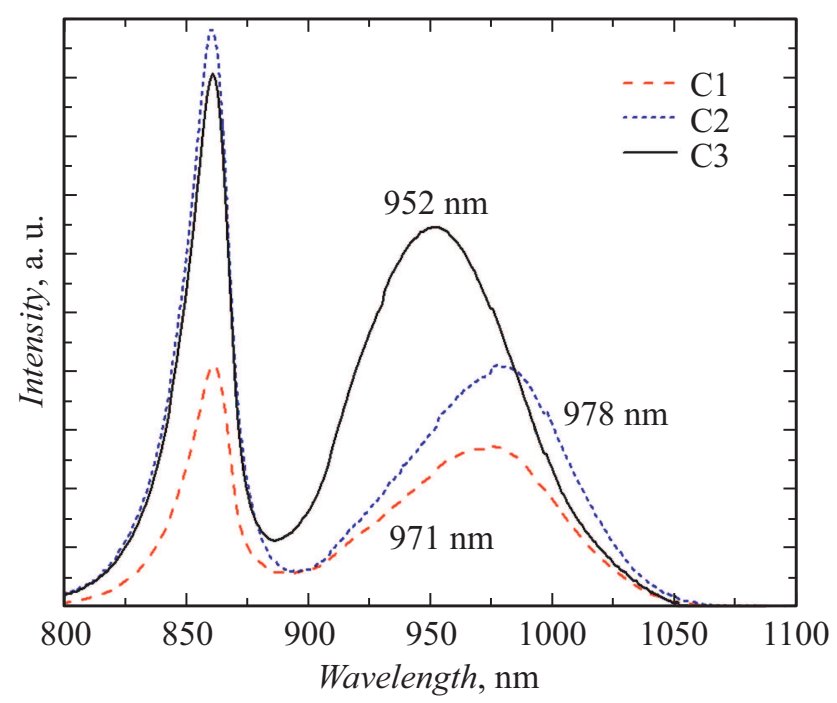

Рис. 2. Спектры ФЛ образцов $\mathrm{C} 1-\mathrm{C} 3$, измеренные в центрах пластин.

пластины уменьшается в 1.25 раза. Ширина пика ФЛ, измеренная на полувысоте (FWHM), составила величину $95 \mathrm{meV}$, что говорит о большом разбросе трехмерных островков по размерам и/или составам. На рис. 2 показаны спектры ФЛ образцов $\mathrm{C} 1-\mathrm{C} 3$, измеренные в центрах пластин. Спектр структуры С2 продемонстрировал длинноволновый сдвиг пика ФЛ, соответствующего излучению от трехмерных островков $\operatorname{InGaP}(\mathrm{As})$, и увеличение интенсивности примерно на $30 \%$ по сравнению с данными для образца С1. Таким образом, увеличение температуры выдержки привело к сдвигу длины волны излучения трехмерных островков на $6 \mathrm{~nm}$. Кроме того, улучшилась однородность массива островков, о чем свидетельствует уменьшение полуширины пика ФЛ до $90 \mathrm{meV}$ и разброса положения пика ФЛ, при измерении по площади пластины на удалении $30 \mathrm{~mm}$ от центра пластины, составившего $5 \mathrm{~nm}$ для образца С2 (981-986 nm) вместо $12 \mathrm{~nm}$ для образца С1 (рис. 1). Изменение технологических режимов формирования для образца С3 привело к коротковолновому сдвигу положения пика ФЛ, соответствующего излучению от трехмерных островков $\operatorname{InGaP}($ As), до $953 \mathrm{~nm}$. При этом интенсивность пика ФЛ оказалась наибольшей, более чем в 2 раза превышающей интенсивность пика ФЛ образца С1. Следует также отметить, что при сравнимой с образцом С2 полушириной пика ФЛ (97 meV) образец С3 продемонстрировал наибольший разброс положения пика ФЛ (в диапазоне 943-976 nm) и интенсивности пика ФЛ (менялась в 2 раза) по площади подложки. Так, интенсивность менялась в 2 раза, а длина волны от 943 до $976 \mathrm{~nm}$ от края до края пластины.

На рис. 3 показано планарное (001) ПЭМ изображение образца $\mathrm{C} 4$, на котором отчетливо видны трехмерные островки. При последовательном обзоре образца в режиме темного поля в рефлексах $g=(2-20)$, (400), (220), (040) было установлено, что трехмерные островки имеют овальную форму, вытянутую вдоль направления [1-10]. При этом они часто выстраиваются в линию „один-за-другим“ вдоль того же направления. При этом вдоль ортогонального направления [110] таких эффектов не наблюдается, т.е. направления $[110]$ и $[1-10]$ не эквивалентны. Очевидно, это связано с анизатропностью скорости поверхностной диффузии во время выдержки слоя InGaP, которая по всей видимости обусловлена направленностью оборванных связей на ростовой поверхности. Оценочная плотность островков составила величину $1.3 \cdot 10^{10} \mathrm{~cm}^{-2}$. Если предположить, что увеличение интенсивности пика ФЛ островков $\operatorname{InGaP}(\mathrm{As})$ в образце С3 связано с увеличением их плотности, то ост-

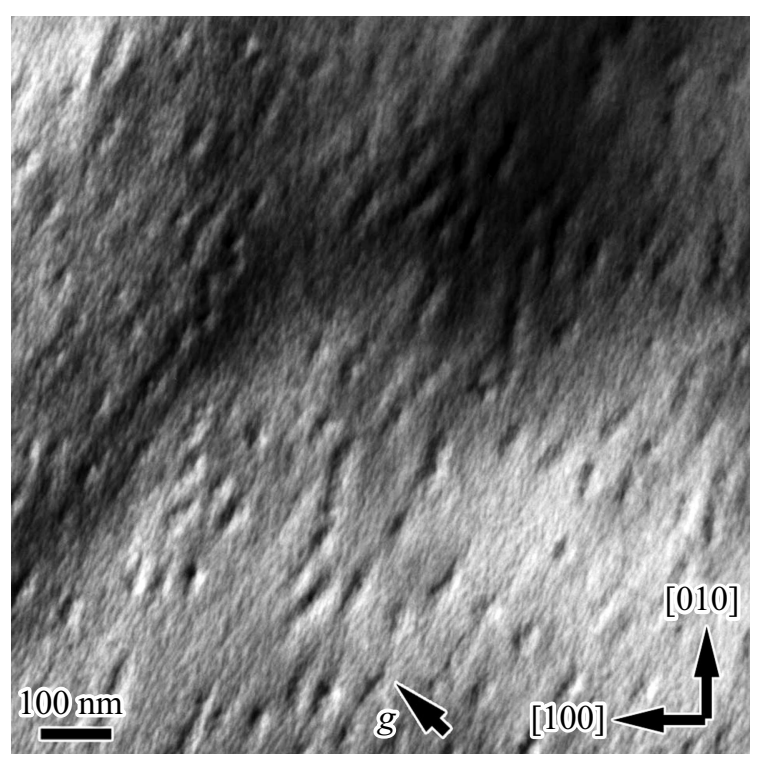

Рис. 3. Темнопольное ПЭМ-изображение планарной поверхности образца (001), полученное в двулучевых условиях в отражении $g=(220)$. 
ровки, сформированные на слое InGaP толщиной $2 \mathrm{~nm}$, будут иметь меньшую плотность.

\section{Заключение}

Продемонстрирована возможность формирования трехмерных низкоплотных квантово-размерных объектов методом замещения элементов пятой группы непосредственно во время эпитаксиального роста. Показано, что при замещении фосфора в тонком слое InGaP на мышьяк формируются трехмерные островки, излучающие в спектральном диапазоне $\sim 1 \mu \mathrm{m}$ при комнатной температуре.

Оценочная плотность островков составила $1.3 \cdot 10^{10} \mathrm{~cm}^{-2}$, что перспективно для возможного применения в системах с нейроморфными вычислениями. Как показано ранее [21], использование трехмерных низкоплотных квантово-размерных объектов позволяет увеличить силу осциллятора, что наряду с низкой плотностью свидетельствует о возможности использования данных трехмерных квантово-размерных объектов для создания источников одиночных фотонов и массивов микро-лазеров.

\section{Финансирование работы}

Работа выполнена при поддержке Министерства науки и высшего образования Российской федерации, проект тематики научных исследований № 2019-1442.

\section{Конфликт интересов}

Авторы заявляют, что у них нет конфликта интересов.

\section{Список литературы}

[1] N. Somaschi, V. Giesz, L. De Santis, J.C. Loredo, M.P. Almeida, G. Hornecker, S.L. Portalupi, T. Grange, C. Antón, J. Demory, C. Gómez, I. Sagnes, N.D. LanzillottiKimura, A. Lemaítre, A. Auffeves, A.G. White, L. Lanco, P. Senellart. Nat. Photonics, 10 (5), 340 (2016). DOI: $10.1038 /$ nphoton.2016.23

[2] M. Schmidt, M.V. Helversen, S. Fischbach, A. Kaganskiy, R. Schmidt, A. Schliwa, T. Heindel, S. Rodt, S. Reitzenstein. Opt. Mater. Express, 10 (1), 76 (2019).

DOI: $10.1364 /$ ome.10.000076

[3] F. Basso Basset, M.B. Rota, C. Schimpf, D. Tedeschi, K.D. Zeuner, S.F. Covre da Silva, M. Reindl, V. Zwiller, K.D. Jöns, A. Rastelli, R. Trotta. Phys. Rev. Lett., 123 (16), 160501 (2019). DOI: 10.1103/physrevlett.123.160501

[4] M. Zopf, R. Keil, Y. Chen, J. Yang, D. Chen, F. Ding, O.G. Schmidt. Phys. Rev. Lett., 123 (16), 160502 (2019). DOI: $10.1103 /$ physrevlett.123.160502

[5] T. Kroh, J. Wolters, A. Ahlrichs, A.W. Schell, A. Thoma, S. Reitzenstein, J.S. Wildmann, E. Zallo, R. Trotta, A. Rastelli, O.G. Schmidt, O. Benson. Sci. Rep., 9(1), 13728 (2019). DOI: $10.1038 / \mathrm{s} 41598-019-50062-\mathrm{x}$
[6] H. Wang, Y.-M. He, T.-H. Chung, H. Hu, Y. Yu, S. Chen, X. Ding, M.-C. Chen, J. Qin, X. Yang, R.-Z. Liu, Z.-C. Duan, J.-P. Li, S. Gerhardt, K. Winkler, J. Jurkat, L.-J. Wang, N. Gregersen, Y.-H. Huo, Q. Dai, S. Yu, S. Höfling, C.-Y. Lu, J.-W. Pan Nat. Photonics, 13 (11), 770-775 (2019). DOI: $10.1038 / \mathrm{s} 41566-019-0494-3$

[7] M. Anderson, T. Müller, J. Huwer, J. Skiba-Szymanska, A.B. Krysa, R.M. Stevenson, J. Heffernan, D.A. Ritchie, A.J. Shields. Npj Quantum Inf., 6(1), 14 (2020). DOI: 10.1038/s41534-020-0249-5

[8] S. Kolatschek, S. Hepp, M. Sartison, M. Jetter, P. Michler, S.L. Portalupi. J. Appl. Phys., 125 (4), 045701 (2019). DOI: $10.1063 / 1.5050344$

[9] M.C. Löbl, S. Scholz, I. Söllner, J. Ritzmann, T. Denneulin, A. Kovács, B.E. Kardynał, A.D. Wieck, A. Ludwig, R.J. Warburton. Commun. Phys., 2 (1), 93 (2019). DOI: 10.1038/s42005-019-0194-9

[10] T. Heuser, J. Grose, S. Holzinger, M.M. Sommer, S. Reitzenstein. IEEE J. Sel. Top. Quantum Electron., 26 (1), 1 (2020). DOI: $10.1109 /$ jstqe.2019.2925968

[11] E.P. Blair, S. Koziol. Proc.: ICRC. 2017. DOI: $10.1109 /$ icrc.2017.8123685

[12] T. Heuser, J. Große, A. Kaganskiy, D. Brunner, S. Reitzenstein. APL Photonics, 3 (11), 116103 (2018). DOI: $10.1063 / 1.5050669$

[13] V.M. Ustinov, N.A. Maleev, A.E. Zhukov, A.R. Kovsh, A.Y. Egorov, A.V. Lunev, B.V. Volovik, I.L. Krestnikov, Y.G. Musikhin, N.A. Bert, P.S. Kop'ev, Z.I. Alferov, N.N. Ledentsov, D. Bimberg. Appl. Phys. Lett., 74 (19), 2815 (1999). DOI: 10.1063/1.124023

[14] P. Yu, W. Langbein, K. Leosson, J.M. Hvam, N.N. Ledentsov, D. Bimberg, V.M. Ustinov, A.Y. Egorov, A.E. Zhukov, A.F. Tsatsul'nikov, Y.G. Musikhin. Phys. Rev. B., 60 (24), 16680 (1999). DOI: 10.1103/physrevb.60.16680

[15] A.R. Kovsh, A.E. Zhukov, D.A. Livshits, A.Y. Egorov, V.M. Ustinov, M.V. Maximov, Yu.G. Musikhin, N.N. Ledentsov, P.S. Kop'ev, Zh.I. Alferov, D. Bimberg. Electron. Lett., 35 (14), 1161 (1999). DOI: $10.1049 / \mathrm{el}: 19990813$

[16] A.S. Shkolnik, L.Y. Karachinsky, N.Y. Gordeev, G.G. Zegrya, V.P. Evtikhiev, S. Pellegrini, G.S. Buller. Appl. Phys. Lett., 86 (21), 211112 (2005). DOI: 10.1063/1.1938000

[17] A. Rantamäki, G.S. Sokolovskii, S.A. Blokhin, V.V. Dudelev, K.K. Soboleva, M.A. Bobrov, A.G. Kuzmenkov, A.P. Vasil'ev, A.G. Gladyshev, N.A. Maleev, V.M. Ustinov, O. Okhotnikov. Opt. Lett., 40 (14), 3400 (2015). DOI: 10.1364/ol.40.003400

[18] Single Semiconductor Quantum Dots, ed. by P. Michler (Heidelberg: Springer-Verlag, 2009) 389 p. DOI: 10.1007/978-3-540-87446-1

[19] E. Schlottmann, D. Schicke, F. Krüger, B. Lingnau, C. Schneider, S. Höfling, K. Lüdge, X. Porte, S. Reitzenstein. Opt. Express., 27 (20), 28816 (2019). DOI: $10.1364 /$ oe. 27.028816

[20] G. Trevisi, L. Seravalli, P. Frigeri, S. Franchi. Nanotechnology, 20 (41), 415607 (2009). DOI: $10.1088 / 0957-4484 / 20 / 41 / 415607$

[21] J.P. Reithmaier, G. Sçk, A. Löffler, C. Hofmann, S. Kuhn, S. Reitzenstein, L.V. Keldysh, V.D. Kulakovskii, T.L. Reinecke, A. Forchel. Nature, 432 (7014), 197 (2004). DOI: 10.1038 /nature02969 